\title{
Coexisting Tertiary Hyperparathyroidism and Severe Hypothyroidism in an End-Stage Renal Disease Patient on Hemodialysis
}

\author{
Yahya Osman Malik, ${ }^{1, *}$ Syed Mohsin Raza, ${ }^{1}$ and Sophia Arunselvan ${ }^{1}$ \\ ${ }^{1}$ Department of Nephrology, School of Medicine, Wayne State University, Detroit, USA \\ "Corresponding author: Yahya Osman Malik, Department of Nephrology, School of Medicine, Wayne State University, Detroit, MI, USA. Tel: +1-3137457145, E-mail: \\ yosman@med.wayne.edu
}

Received 2015 January 04; Accepted 2015 January 26.

\begin{abstract}
Introduction: The clinical syndrome of uremia is a bedside diagnosis which mimics a wide spectrum of other clinical disorders, most commonly thyroid disease. End-stage renal disease (ESRD), as a disorder, frequently alters thyroid hormone metabolism, and this is not significantly normalized by dialysis. Although the thyroid and parathyroid glands are considered independent organs, their anatomical juxtaposition in the neck, as well as sharing a common embryological origin, might play a role in some of the possible association between thyroid and parathyroid disease. It has been demonstrated in experimental animals that changes in the thyroid gland might lead to pathological changes in the parathyroids and vice versa.

Case Presentation: An incidence of as high as $25 \%$ of hypothyroidism has been reported in patients with ESRD on dialysis. We report a patient with ESRD on maintenance hemodialysis (MHDx) who has had a combination of profound tertiary hyperparathyroidism (HPTH) and severe hypothyroidism.

Conclusions: Literature search revealed an increased prevalence of hypothyroidism with secondary HPTH from renal failure. Although there is increased prevalence of hypothyroid state in secondary HPTH from renal failure, the association appears much weaker in primary HPTH and again no conclusive pathological relation has been identified between the two endocrine glands. A closer look and perhaps long-term prospective studies are required in the future to determine this association.
\end{abstract}

Keywords: Parathyroid Hormone, Hypothyroidism, Hyperparathyroidism, End-Stage Renal Disease, Hemodialysis

\section{Introduction}

Co-existence of a wide spectrum of thyroid and parathyroid disorders has been widely reported in the past. Secondary hyperparathyroidism is one of the most common abnormalities in mineral metabolism in dialysis patients. There is well studied association of increased morbidity and mortality due to disturbed mineral metabolism in End-Stage Renal Disease (ESRD) patients on dialysis (1). On the contrary, thyroid disorders in ESRD patients have attracted less attention despite new growing evidence of a higher mortality in ESRD patients with thyroid disease, in particular hypothyroidism. A plethora of retrospective studies, predominantly from surgical literature, found an association between different thyroid disorders (nodules, goiter, malignancies, and hypothyroidism) and primary as well as secondary hyperparathyroidism (HPTH) (2-4). However, most of those studies have failed to prove a causal, or a temporal relationship in the pathological findings in the two juxtaposed endocrine organs. So far, no conclusive pathological relation has been identified between the two endocrine glands.

\section{Case Presentation}

We report a 62-year-old black female with known ESRD on MHDx for eight years. Other co-morbidities include hypertension, bronchial asthma, coronary artery disease, and recurrent transient ischemic attacks. She was admitted to the hospital after sustaining right-sided hip fracture related to an accidental fall. Review of systems was positive for fatigue, generalized weakness, constipation, and cold intolerance. Vitals signs: pulse rate 54/bpm, BP 117/69 $\mathrm{mmHg}$, and respiratory rate $16 /$ minute.

On physical exam, patient seemed very slow in response and was quite bradykinetic. Face was puffy with cold, dry skin and coarse hair. Her right leg was externally rotated. Neck examination revealed a homogenous, firm mass $(4 \times 4 \mathrm{~cm})$ which moved with swallowing and was not tethered to the skin (Figure 1 ).

Neurological exam revealed delayed relaxation phase of ankle jerk. Laboratory values: serum calcium $8.4 \mathrm{mg} / \mathrm{dL}$, serum albumin $2.5 \mathrm{gm} / \mathrm{dL}$, serum magnesium $1.5 \mathrm{mg} / \mathrm{dL}$, serum phosphorous $3.9 \mathrm{mg} / \mathrm{dL}$, intact parathyroid hormone (iPTH) $6619 \mathrm{pg} / \mathrm{mL}$, thyroid stimulating hormone (TSH) $454 \mathrm{IU} / \mathrm{mL}$, free thyroxin $0.2 \mathrm{ng} / \mathrm{dL}$, and 25 hydroxyvitamin D level $12 \mathrm{ng} / \mathrm{mL}$. Her serum inorganic iodide 
level was undetectable. X-ray of right hip revealed femoral neck fracture. Ultrasound of the neck showed diffuse heterogeneous and nodular enlargement of the entire thyroid with multiple tiny cysts and nodular changes with a complex hypo echoic nodule in the isthmus measuring $(2.1 \times 1.2 \times 1.2 \mathrm{~cm})$. Following a diagnosis of tertiary hyperparathyroidism and severe hypothyroidism, associated with a right-sided femoral neck fracture, she underwent a total hip arthroplasty followed the next day by subtotal parathyroidectomy and right thyroid lobectomy. Due to concerns for hungry bone syndrome and her significant hypothyroid state postoperatively, she was closely monitored in intensive care with an endocrinology consultation on board.

Post operatively, her labs revealed iPTH $112 \mathrm{pg} / \mathrm{mL}$ and calcium $5.9 \mathrm{mg} / \mathrm{dL}$. But she remained asymptomatic. She was treated with intravenous (IV) calcium gluconate in combination with Calcitriol 5 micrograms (mcg) IV twice a day for two days and then switched to oral Calcitriol 0.5 mcg daily. She also started on $25 \mathrm{mcg}$ of IV levothyroxine, initially for four days, and then switched to levothyroxine 75 mcg daily, orally.

Her thyroid histology was consistent with Hashimoto thyroiditis with diffuse infiltration by lymphocytes; plasma cells; and multiple lymphoid follicles, some with germinal centers; along with atrophic follicles with sparse colloid and lined by flattened epithelium or oncocytic (Hürthle cell) change. In addition, some areas of the thyroid parenchyma were replaced by fibrous tissue. Her parathyroid histology showed hyperplastic parathyroid tissues.

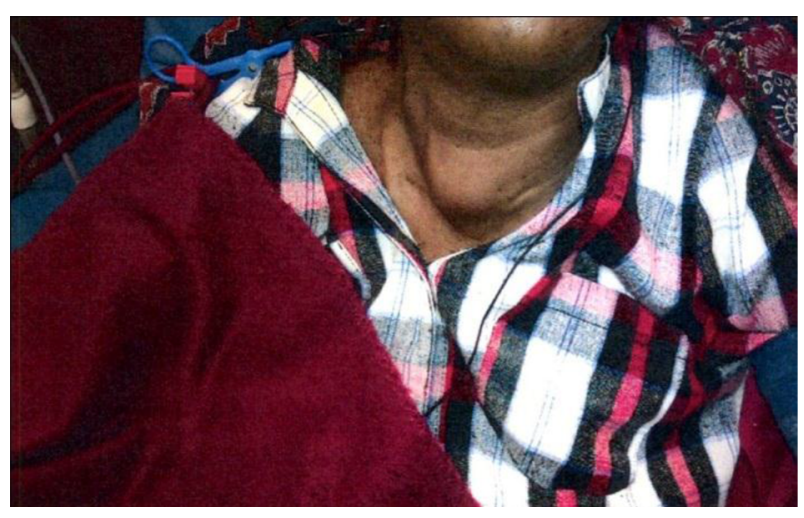

Figure 1. View of Grossly Enlarged Parathyroid Glands

\section{Discussion}

The possible relation between thyroid and parathyroid disease was addressed almost a century ago by Tan-

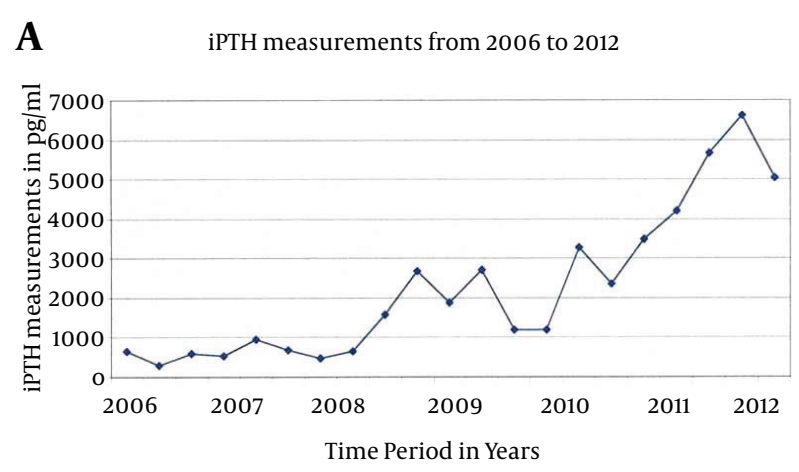

B

TSH measurements from 2006 to 2012

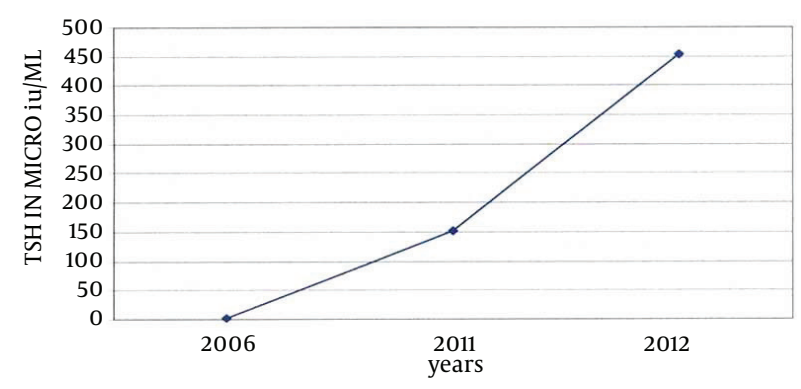

Figure 2. A) iPTH levels over a seven-year period, B) TSH levels over a seven-year period

berg (5) who has carried out elegant studies in cats with non-specific changes in both glands, but no definitive conclusion could be reached. Three decades later, Kissin et al. reported a case of associated myxedema and hyperparathyroidism (6). The prevalence and incidence of nodular goiter and hypothyroidism is much higher in ESRD patients on MHDx, especially female patients, compared to the general population $(7,8)$ Many studies reported higher incidence of nodular goiter and hypothyroidism in ESRD in the range of $40-60 \%$ and $3-10 \%$, respectively $(7,9$, 10). The diagnosis of hypothyroidism is often missed in ESRD patients. Clinical diagnosis can be quite challenging as its symptoms and signs resemble, or are masked by, those of clinical uremia. Secondly, the thyroid lab profile of uremic patients without any clinical thyroid disease can be quite confusing due to altered thyroid functions (low TT3, TT4, and free T4 index with mild elevations of TSH of up to $10 \mu \mathrm{U} / \mathrm{mL}$ ). However, a TSH $>20 \mu \mathrm{U} / \mathrm{mL}$ was found to be a reliable biochemical confirmatory test in ESRD patients with hypothyroidism $(9,11)$. Although numerous contributing factors to the development of hypothyroidism in ESRD patients have been proposed, the exact underlying mechanisms linking renal and thyroid dysfunction remain unclear. Overall, the predisposing factors to thyroid disorders in this patient's population are 
thought to be multi-factorial including iodine exposure during the dialysis session, inorganic iodide accumulation leading to formation of nodular goiter, eventual development of hypothyroidism, concomitant auto-immune disorders, medications such as heparin and furosemide, and calcium accumulation from dialysate. Additional factors that might contribute to the increased incidence of thyroid disease in renal failure patients include possible excessive calcitonin production and ill-defined uremic toxins acting as goitrogens, in addition to older age, diabetes history (which might impair the thyroid capacity to produce adequate hormones in ESRD), and genetic predisposition $(9,11)$.

Most of the literature published in the past has focused on parathyroid/thyroid surgeries with co-existing thyroid/parathyroid abnormalities coincidentally reported at the time of surgery $(2,4)$. However, the temporal sequence of the two organs' dysfunction is lacking, eliminating any element of closer scrutiny of a possible pathophysiological relation between HPTH and thyroid disease in ESRD patients. Walker et al. (12) demonstrated in experimental animals a relation between hypothyroidism and subsequent development of hyperparathyroidism. This has been preceded long ago by a similar study in the 1940s by Malcolm, et al. (13) who had similar findings, suggestive of a common denominator between thyroid disorders and HPTH which could well be a state of subclinical or subtle hypothyroidism.

In the case of our patient, who has a long vintage on dialysis coupled with poor compliance history, it will be difficult to assume that hypothyroidism has precipitated this magnitude of tertiary HPTH. However, the concomitant massive elevations of both PTH and TSH deserves closer attention as to whether this is purely coincidental with the two disorders progressing simultaneously side by side, or if there is a possible unknown interaction. Therefore, an important question needs to be raised in this context: Is the tight anatomical proximity an adequate explanation or is there an actual physiological interaction between the two juxtaposed endocrine organs? In addition to the well-known standard clinical factors predisposing ESRD patients to severe HPTH (hypocalcaemia, hypophosphatemia, etc.), has her severe hypothyroidism played any role in causing HPTH of that severity? On the other hand, though biopsy suggests Hashimoto thyroiditis, could there be other possible contributing factors including iodine deficiency or compression atrophy by the massively enlarged parathyroid gland. So far, there are no other reported cases of profound hypothyroidism and tertiary hyperparathyroidism. Our patient has shown the most concomitant elevation of PTH and TSH (Figure 2, A and B), and survived two major surgeries quite well.
The exact underlying pathophysiology linking renal failure and thyroid dysfunction remains unclear. There are several published data showing coexisting hypothyroidism with primary hyperparathyroidism. Combined primary thyroid and parathyroid pathologies can occur in endocrine neoplasia type 2 syndromes, lithium therapy, and radiation exposure-none of which are present in our patient. Literature search revealed an increased prevalence of hypothyroidism with secondary HPTH from renal failure. Although there is increased prevalence of hypothyroid state in secondary HPTH from renal failure, the association appears much weaker in primary HPTH, and again, no conclusive pathological relation has been identified between the two endocrine glands. So far, no published reports are available for combined tertiary HPTH with hypothyroidism, especially in a patient like ours who had significant hormonal abnormalities. This clinical scenario creates a challenge in clinical decision making and management. A closer look, and perhaps long-term prospective studies, is required in the future to determine this association.

\section{Footnote}

Authors' Contributions: Yahya Osman, senior author, nephrology teaching faculty-supervised other two authors in writing the case presentation, reviewed the clinical findings, and edited entire manuscript before finalization and submission. Sophia Arunselvan, nephrology fellowcollected clinical data on the patient and wrote the initial case. Syed Mohsin Raza, nephrology fellow-assisted Dr. Arunselvan on collection of data and writing of initial presentation.

\section{References}

1. Block GA, Klassen PS, Lazarus JM, Ofsthun N, Lowrie EG, Chertow GM. Mineral metabolism, mortality, and morbidity in maintenance hemodialysis. J Am Soc Nephrol. 2004;15(8):2208-18. doi: 10.1097/01.ASN.0000133041.27682.A2. [PubMed:15284307].

2. Sidhu S, Campbell P. Thyroid pathology associated with primary hyperparathyroidism. Aust N Z J Surg. 2000;70(4):285-7. [PubMed: 10779061].

3. dell'Erba L, Baldari S, Borsato N, Bruno G, Calo-Gabrieli G, Carletto $\mathrm{M}$, et al. Retrospective analysis of the association of nodular goiter with primary and secondary hyperparathyroidism. Eur J Endocrinol. 2001;145(4):429-34. [PubMed: 11581000].

4. Sianesi M, Del Rio P, Arcuri MF, Iapichino G, Giuseppe R. Hyperparathyroidism Associated With Thyroid Pathology. Am J Surg. 2003;185(1):58-60.

5. Tanberg A. The Relation between the Thyroid and Parathyroid Glands. J Exp Med. 1916;24(5):547-59. [PubMed: 19868059].

6. Kissin M, Bakst H. Co-existing myxedema and hyperparathyroidism; case report. J Clin Endocrinol Metab. 1947;7(2):152-8. doi: 10.1210/jcem7-2-152. [PubMed: 20240745]. 
7. Kaptein EM, Quion-Verde H, Chooljian CJ, Tang WW, Friedman PE, Rodriquez HJ, et al. The thyroid in end-stage renal disease. Medicine (Baltimore). 1988;67(3):187-97. [PubMed: 3259281].

8. Kaptein EM. Thyroid hormone metabolism and thyroid diseases in chronic renal failure. Endocr Rev. 1996;17(1):45-63. doi: 10.1210/edrv-171-45. [PubMed: 8641223].

9. Lin CC, Chen TW, Ng YY, Chou YH, Yang WC. Thyroid dysfunction and nodular goiter in hemodialysis and peritoneal dialysis patients. Perit Dial Int. 1998;18(5):516-21. [PubMed: 9848631].

10. Miki H, Oshimo K, Inoue H, Kawano M, Tanaka K, Komaki K, et al. Thyroid nodules in female uremic patients on maintenance hemodialy- sis. J Surg Oncol. 1993;54(4):216-8. [PubMed: 8255080].

11. Tang WW, Kaptein EM, Massry SG. Diagnosis of hypothyroidism in patients with end-stage renal disease. Am J Nephrol. 1987;7(3):192-7. [PubMed: 3631149].

12. Walker RP, Oslapas R, Ernst K, Hessel P, Nayyar R, Lawrence AM, et al. Hyperparathyroidism induced by hypothyroidism. Laryngoscope. 1993;103(3):263-8. doi: 10.1288/00005537-199303000-00005. [PubMed: 8441313].

13. Malcolm J, Griesbach WE. Hyperplasia of the parathyroids associated with osteitis fibrosa in rats treated with thiouracil and related compounds. BrJ Exp Pathol. 1949;30(1):17-23. [PubMed:18128092]. 